\title{
La reconstruction sartrienne de la vie de Flaubert
}

\section{Young-Rae Ji}

\section{OpenEdition}

Journals

Édition électronique

URL : http://journals.openedition.org/recherchestravaux/221

DOI : 10.4000/recherchestravaux.221

ISSN : 1969-6434

\section{Éditeur}

UGA Éditions/Université Grenoble Alpes

\section{Édition imprimée}

Date de publication : 15 octobre 2007

Pagination : 49-64

ISBN : $978-2-84310-111-3$

ISSN : 0151-1874

\section{Référence électronique}

Young-Rae Ji, « La reconstruction sartrienne de la vie de Flaubert », Recherches \& Travaux [En ligne], 71 | 2007, mis en ligne le 15 avril 2009, consulté le 08 septembre 2020. URL : http:// journals.openedition.org/recherchestravaux/221 ; DOI : https://doi.org/10.4000/recherchestravaux 221 


\section{La reconstruction sartrienne de la vie de Flaubert}

L'Idiot de la famille ${ }^{\mathrm{I}}$ concerne deux écrivains : Sartre et Flaubert. Pourtant l'accueil de l'œuvre de ces deux côtés est fort divergent. Contrairement aux sartriens qui ont reçu chaleureusement cet ultime ouvrage de Sartre en le louant comme " un livre des merveilles" » ou "le seul roman possible aujourd'hui ${ }^{3}$ ", les flaubertiens l'ont accueilli avec froideur : ils ont préféré garder le silence ou, s'ils ont eu l'occasion d'exprimer leurs observations, ils n'ont pas ménagé ce « Flaubert » sartrien. Même s’ils estiment « la beauté » de cet ouvrage, ils ne le considèrent pas comme un travail sérieux sur Flaubert. Surtout, ils mettent en doute l'authenticité de la documentation dont Sartre s'est servi, et dénoncent les erreurs et les inventions qui « fourmillent ${ }^{4} »$ dans son livre.

Est-ce qu'il y a vraiment tant d'erreurs dans celui-ci ? Est-ce que ce «Flaubert » est tellement faussé par Sartre, pour être si gênant aux yeux des flaubertiens ? Nous essayerons, dans cette étude, de comprendre ces réactions des flaubertiens en examinant nous-mêmes L'Idiot de la famille dans une optique plus positiviste ou plus universitaire, c'est-à-dire que nous entreprendrons d'interroger sa véracité biographique en dégageant les éléments qui ne correspondent pas aux faits considérés comme vrais, ou en décelant les

I. J.-P. Sartre, L'Idiot de la famille: Gustave Flaubert de I82I à I857, nouvelle édition revue et complétée, Gallimard, « Bibliothèque de philosophie », I988, tomes I et II (désormais abrégé en $I D F)$.

2. Serge Doubrovsky, «Une étrange toupie », Le Monde des livres, 2 juillet I 97 I.

3. Michel Rybalka, «Comment peut-on être Flaubert? ", Le Nouvel Observateur, 17 mai I 97 I, p. 55 .

4. Jean Bruneau, "L'intervention », Langages de Flaubert, Actes du colloque de London (Canada, 1973), Minard, I976, p. 230. 
erreurs commises par Sartre, non seulement au moment de la documentation, mais aussi au moment de l'établissement de ses hypothèses. Ce faisant, nous pourrons mieux comprendre la déclaration inattendue de Sartre qui nous invite à lire son Flaubert " comme un roman' ", et mettre en valeur plus objectivement les mérites authentiques de cette œuvre-somme.

Si L'Idiot de la famille, malgré la documentation très studieuse de Sartre, ne nous donne pas l'impression d'un travail académique et soigneusement vérifié, c'est qu'il y a, dans la construction et dans la présentation de cet ouvrage, plusieurs défauts à différents niveaux.

Premièrement, la raison pour laquelle les lecteurs de L'Idiot de la famille ressentent sa documentation comme non rigoureuse est d'abord l'absence de référence pour beaucoup de citations. Non seulement la plupart des phrases citées des œuvres de jeunesse de Flaubert ne s'accompagnent pas d'indications, mais aussi, pour ses autres œuvres, les renseignements de la référence sont assez flous sauf quand il s'agit de la correspondance. Par ailleurs, même si Sartre fournit des notes en bas de page, celles-ci ne nous livrent que le minimum des informations ${ }^{6}$ (parfois fausses ${ }^{7}$ ), de telle sorte que, faute d'une bibliographie établie par Sartre lui-même dans L'Idiot de la famille, nous devons recourir à d'autres sources bibliographiques pour les confirmer. En outre, les phrases citées à l'intérieur du texte sont souvent inexactes. Elles ne correspondent pas tout à fait aux textes originaux, parce que Sartre les a maniées librement pour les adapter dans son contexte, sans grand souci de leur exactitude $^{8}$. Normalement, cela ne gênera pas beaucoup les lecteurs ordinaires, mais il est certain que, pour un lecteur exigeant et selon certains points de vue, même les légères erreurs peuvent être considérées comme inadmissibles.

Deuxièmement, il faut signaler la lourdeur de la forme de présentation de L'Idiot de la famille. Quand ses trois tomes furent publiés en I97 I et I972, les

5. J.-P. Sartre, Situations IX, Gallimard, 1972, p. I 23 ; J.-P. Sartre, Situations X, Gallimard, I976, p. 94.

6. Voir par exemple IDF, t. I, p. 884, note I. On lit : «Éd. Charpentier, p. 42-1 2 » au lieu de : « Flaubert, Premières cuvres, t. I, Éd. Eugène Fasquelle, "Bibliothèque-Charpentier », I9I 3 , p. 42 -IO2», etc.

7. Voici quelques exemples parmi d'autres. IDF, t. I, p. 444 : la lettre à Ernest Chevalier est du 24 mars I 837 , non du 24 juin I 837 ; IDF, t. I, p. 624 : dans la note $\mathrm{I}$, la seconde citation de la lettre à Louise Colet est d'une lettre du 24 avril I 852 , et dans la note 2, la première lettre à Louis Bouilhet est du i9 décembre i 850 , non du 9 décembre I 850.

8. Voir par exemple IDF, t. I, p. 617. Dans cette page, Sartre ne nous donne qu'une seule référence, dans la note I : «Théâtre. Éd. Conard, p. 253-254». Cependant nous trouvons en fait trois phrases citées, c'est-à-dire deux autres citations de plus, puisées dans des pages différentes. Et Sartre les a adaptées librement en modifiant à son gré les textes originaux. 
premières impressions des lecteurs devant cet ouvrage « qui ressemble au

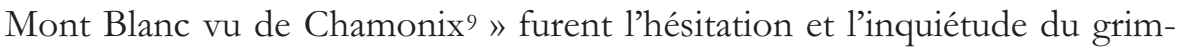
peur. Pour à peu près trois mille pages serrées en grand format, on ne voyait aucune information extérieure, sauf une table des matières constituant un guide peu utilisable. On mit en question leur lisibilité même ${ }^{10}$. Tenant compte de cette difficulté de la lecture, l'éditeur de la nouvelle édition de 1988 a ajouté deux index assez complets : «l'index des noms propres » et «l'index bibliographique ». Mais la table des matières garde toujours ses défauts initiaux dans cette nouvelle édition. Tantôt elle manque de cohérence dans sa présentation ${ }^{11}$, tantôt elle ne correspond pas au texte ${ }^{12}$. Ce genre de négligence et de disproportion nous donne l'impression générale qu'il s'agit d'une table des matières à l'état de brouillon, non à l'état définitif. Il nous semble que cette sorte de déficiences ne sont pas intervenues au moment où un éditeur quelconque a établi cette table des matières, mais que Sartre lui-même n'a pas eu le temps de «faire court», qu'il n'a pas pu débarrasser son livre des fautes apparentes ou perfectionner son texte. Cela montre que c'est plutôt comme écrivain que comme spécialiste que Sartre interprète la destinée de Flaubert dans L'Idiot de la famille.

Troisièmement, nous remarquons que les raisonnements développés dans L'Idiot de la famille sont fondés sur des faits fictifs de la vie de Flaubert, ce qui est le plus grave défaut. Sur ce sujet, les flaubertiens comme Jean Bruneau ont déjà refusé de souscrire aux nombreuses thèses de Sartre concernant les faits historiques sur les membres de la famille Flaubert ${ }^{13}$. J. Bruneau a même relevé, après la mort de Sartre, plusieurs points qu'il ne pouvait pas admettre en tant

9. Maurice Nadeau, «Sartre et l'Idiot de la famille », La Quinzaine littéraire, n I I9, du rer au I 5 juin I97I, p. 3 .

ı. Voir Claude Burgelin, «Lire "L'Idiot de la famille" ? ", Littérature, n 6, mai I 972, p. I I I I 2 .

I I. Voir par exemple la table des matières pour le chapitre VII du livre III de la deuxième partie (IDF, t. II, p. 2169).

I 2. Par exemple, la table des matières pour la section B du chapitre VI du livre II de la deuxième partie (IDF, t. II, p. 2 I 68) devrait être corrigée comme suit :

B. - L'bistoire (le psychodrame) I 337

La Contre-révolution [1 343]

I. Le reflux I 349

2. La prise de conscience impossible 1353

Passage à l'imaginaire [ 1367 ]

La vengeance des aristocrates [1 392]

Les Enragés [1423]

Le sérieux du comique [1442]

Le psychodrame [1 450 ]

Le consentement ou comique du sérieux [1 464]

I 3. Voir Langages de Flaubert, éd. citée, p. 230. 
que «Flaubertiste ${ }^{14} »$. Cependant nous trouvons, dans ses arguments, presque autant d'ambiguïté que dans ceux de Sartre et nous jugeons que les démonstrations de J. Bruneau ne se jouent pas sur un terrain évident et irréfutable. Cela ne veut pas dire bien sûr que nous plaidions pour la véracité des thèses de Sartre. Au contraire, nous sommes tout à fait d'accord avec les flaubertiens qui prétendent que les thèses de Sartre sont fictives et qu'elles reposent plutôt sur sa propre enfance que sur celle de Flaubert. Mais nous voulons le montrer par une autre voie en tentant de chercher la faille logique à l'intérieur même de ces thèses. Il y a des défauts plus graves que les flaubertiens n'ont pas fait suffisamment remarquer. C'est ce que nous allons montrer maintenant.

Les arguments que Sartre a développés dans L'Idiot de la famille se résument en un mot dans le titre même de cet ouvrage: Gustave était l'idiot de la famille, c'est-à-dire que Gustave est devenu Flaubert, le plus grand romancier français de la seconde moitié du XIX ${ }^{e}$ siècle, parce qu'il était considéré dans son enfance comme « un idiot» au sein de sa famille. Et il y a un événement décisif, selon Sartre, dans la constitution du caractère de Gustave Flaubert : il ne savait pas lire à sept ans. Avant cet événement il y avait «l'âge d'or »; après celuici, ce fut « la Chute». Tout a commencé à sept ans ${ }^{15}$ : « La disgrâce, vers sept ans, a été indubitablement un véritable traumatisme ; à cet âge s'est formée en lui cette "fêlure" qui, de toute manière, le promettait à l'exil, aux "léthargiques mélancolies" » (IDF, t. I, p. 4IO). Passant dès lors pour un demeuré aux yeux d'un père éminent et exigeant, par comparaison avec un frère aîné aux brillantes études et sans histoire, Flaubert serait resté toute sa vie «l'idiot de la famille », à la fois ennemi et prisonnier de cette famille, dont il ne parviendra jamais ni à s'évader ni à se venger.

Dans ce livre, nous trouvons deux piliers de l'argumentation, deux «plannings» (selon l'expression de Sartre) qui soutiennent l'ensemble de la structure logique des arguments de Sartre. Le premier est le «planning familial ${ }^{16}$ » des parents de Gustave, qui déterminent la protohistoire de leur fils cadet en constituant irrémédiablement sa passivité ; le second est le "planning névrotique $^{17}$ » que Gustave intériorise en tant que mécanisme de défense contre le mal de sa vie, et qui sera accompli par sa fameuse crise pendant une nuit de janvier I 844 . Ne pas savoir lire à sept ans est, dans cette logique de Sartre, un événement crucial qui sépare la vie de Gustave en deux : la première moitié

I4. Jean Bruneau, «Jean-Paul Sartre, biographe de Flaubert», Lectures de Sartre, Lyon, PUL, I986, p. I67-I75.

I 5. Voir IDF, t. I, p. I 87, 336, 337, 370, 460, 543, etc.

16. IDF, t. I, p. I 29 et passim.

17. IDF, t. I, p. I 87 et passim. 
prédéterminée par le «planning familial » et la seconde moitié soumise au «planning névrotique ». Toutes les analyses sartriennes sur Flaubert sont fondées sur ces deux hypothèses. Examinons ces deux plannings l'un après l'autre et nous verrons plus clairement le caractère fictif des arguments de Sartre.

Examinons d'abord le «planning familial » qui amène le cadet jusqu'à la crise de l'alphabétisation. Il faut commencer par cette thèse principale : Gustave est considéré comme l'idiot de la famille parce qu'il ne savait pas lire à sept ans et qu'on le compare défavorablement à son aîné Achille. Première question : d'où viennent ces difficultés pour apprendre à lire ? D'après Sartre, c'est « la constitution passive » (IDF, t. I, p. 667) de Gustave qui l'empêche de s'insérer dans l'univers linguistique. À cause de la passivité dans laquelle Gustave est enfermé dès sa protohistoire, le langage restera pour lui une réalité extérieure et quasi étrangère. Pour l'enfant passif et incapable d'accomplir un acte d'affirmation, les mots gardent toujours leur épaisse matérialité et restent comme de « petites pierres sonores » (IDF, t. I, p. 62 I). Par conséquent, « la phrase n'est pas dissoute en lui, elle ne s'efface pas devant la chose dite ou le parleur qui la dit : l'enfant la comprend sans pouvoir l'assimiler » (IDF, t. I, p. 22).

Une deuxième question se pose alors : pourquoi Gustave est-il devenu un enfant passif ? Dans cette analyse de la passivité de Flaubert où certains critiques ont vu la grande originalité de Sartre ${ }^{18}$, nous trouvons autant d'éléments fictifs. La réponse de Sartre est formelle : la surprotection sans amour de sa mère Caroline Flaubert l'a constitué passif. C'est « le zèle pieux et glacial de sa mère qui a constitué Gustave comme agent passif» (IDF, t. I, p. 179) en le laissant, « faute d'aimer ou, peut-être, d'extérioriser son amour [...] comme un poisson sur le sable, vivant sans raison de vivre » (IDF, t. I, p. 333). La froide surprotection qui s'exerçait dès la période d'allaitement de Gustave a empêché ses besoins de se constituer en agression contre l'autre.

Passons maintenant à la troisième question qui se situe à l'origine de toutes les analyses de Sartre : si la passivité de Gustave qui a occasionné en lui le « mauvais rapport initial au langage » (IDF, t. I, p. I 8) était ainsi causée par la froide surprotection de sa mère, pour quelle raison était-elle si froide à l'égard de son fils cadet ? Sartre nous renvoie sur ce point à l'histoire personnelle de Caroline Flaubert. Étant donné qu'il y a peu de sources historiques, Sartre reconstruit ou " imagine ${ }^{19}$ " la personnalité de Mme Flaubert à partir des informations, minces et douteuses, fournies par René Dumesnil dans son Gustave Flaubert, l'homme et l'cuvre.

I 8. Voir Michel Rybalka, «Comment peut-on être Flaubert? ?, art. cité, p. 54. i9. IDF, t. I, p. II6, II9, I 25 , I 35 , etc. 
Voici la version sartrienne de la vie de la mère de Gustave Flaubert (voir $I D F$, t. I, p. 8 I-Io I et p. I 29-I 35) : Caroline Flaubert, fille d'un médecin installé à Pont-l'Évêque, a «l'enfance la plus triste », parce qu'elle a perdu sa jeune mère à sa naissance et qu'elle a également perdu, en conséquence, l'amour de son père, le docteur Fleuriot, mort à son tour dix ans plus tard. Tout en étant élevée par deux dames de Saint-Cyr, puis envoyée chez le docteur Laumonier à Rouen après la mort de celles-ci, Caroline Flaubert grave dans son cœur ses futures exigences : « elle n'épouserait que son père». À l'âge de dix-huit ans, son rêve se réalise lorsqu'elle se marie avec un jeune prévôt d'anatomie, Achille-Cléophas Flaubert, qu'elle considère comme « son père ressuscité » et grâce auquel elle « revenait en arrière et recommençait sa vie à l'âge de dix ans ». Elle aime Achille-Cléophas davantage comme père que comme époux. Et si elle aime ses enfants, c'est qu'elle peut, à travers eux, apprécier « la fécondité du géniteur ». Donc, pour elle, les enfants n'ont d'autre rôle que « de la mettre en possession de sa fonction maternelle », surtout s'agissant des garçons. Cependant, elle veut accoucher d'une fille, enfant de son sexe avec qui elle tenterait de parvenir à « réaliser une version améliorée » de sa propre enfance frustrée, à « limer les griffes de souvenirs encore déchirants ». Par malheur pour elle, le docteur Flaubert ne lui fait que cinq garçons pendant les treize ans qui suivent leur mariage, et dont elle n'accueille avec plaisir que le premier. Dès la deuxième grossesse, elle commence à attendre une fille et « il y eut quatre déceptions ». C’est la raison pour laquelle elle est étrangement indifférente aux décès de ses enfants et, après l'aménagement à l'Hôtel-Dieu, qu'elle accouche de trois enfants en moins de quatre ans jusqu'à la naissance d'une nouvelle Caroline. Bref, Gustave ne fut pas «l'enfant attendu », il fut plutôt un « intrus » ou « une bête étrangère » pour sa mère. Par conséquent, Mme Flaubert devint «mère par devoir » pour son fils cadet et elle exerça sur l'enfant une « surprotection » cachant « un abandon ». C'est l'origine de la passivité de Gustave, qui provoquera chez lui la difficulté de s'insérer dans l'univers linguistique.

Concernant cette thèse, Sartre lui-même avoue que « rien ne prouve qu'il en fut ainsi » (IDF, t. I, p. I 38$)$, mais il nous semble qu'il était convaincu de disposer de faits positifs ${ }^{20}$, et pensait affabuler légitimement sa thèse ainsi. En fait Sartre tente d'étayer toute sa thèse sur la protohistoire de Gustave par l'analyse du "planning familial » des parents de Gustave, c'est-à-dire par le raisonnement fondé sur les dates et les lieux de naissance des enfants dans la famille Flaubert. Quelques informations importantes de l'histoire familiale

20. Voir IDF, t. I, p. 60: « des caractères objectifs de cette cellule... », et p. I77 : «L'origine est objective...». 
des Flaubert données par R. Dumesnil jouèrent un rôle décisif, le rôle d'une sorte de clef de voûte qui justifie toutes les hypothèses de Sartre et qui, par conséquent, soutient l'ensemble de l'architecture de L'Idiot de la famille.

Or nous allons montrer que ces données objectives auxquelles Sartre a fait confiance, au point de les prendre pour pierre angulaire de sa thèse, sont fausses. Les raisonnements de Sartre sur la personne de Mme Flaubert, nous semble-t-il, se développent à partir d'un témoignage de Caroline Commanville, nièce de Gustave Flaubert, qui relate un souvenir partagé avec sa grand-mère. Il s'agit de la nostalgie de Mme Flaubert à l'égard de son ancienne demeure, où elle passa ses premières années de mariage avant le déménagement à l'Hôtel-Dieu :

Le ménage s'établit dans la rue du Petit-Salut $[\ldots]$. Dans mon enfance, grand'mère m'y faisait souvent passer et en regardant les fenêtres elle me disait d’une voix grave, presque religieuse : «Vois-tu, là se sont passées les meilleures années de ma vie $»^{21}$.

Selon les biographes de Flaubert, les parents de Gustave se marièrent le Io février I 8 I 2 et s'installèrent dans une petite maison au 8 de la rue du PetitSalut. C'est là que leur fils aîné, Achille Flaubert, naquit le 9 février I 8 I 3 . Ils y habitèrent pendant six ans avant d'aller loger à la résidence de l'Hôtel-Dieu de Rouen, au début de I 8 I 8, après la mort du docteur Laumonier, ancien chirurgien en chef qui l'avait occupée précédemment. À partir de là, les sources dont Sartre a disposé pour élaborer L'Idiot de la famille ne correspondent plus à celles des flaubertiens. Les informations principales de Sartre proviennent du texte de René Dumesnil, qui comporte lui-même un certain nombre d'erreurs :

Le mariage fut célébré le io février i 8 I 2. [...] Le ménage s'établit rue du PetitSalut. [...] La maison portait le numéro 8. C'est là que naquit le 9 février $\mathrm{I}_{8} \mathrm{I} 3 \mathrm{le}$ premier enfant des Flaubert. [...] Ce fut lui qui succéda à son père. À la mort de Laumonier, sept ans après leur mariage, les Flaubert vinrent habiter l'aile de l'Hôtel-Dieu qui servait de logement au chirurgien en chef. Deux autres enfants qu'ils avaient eus étaient morts en bas âge. C'est le I 2 décembre i 82 I que Gustave est né. [...] Après Gustave, naquirent encore un garçon qui mourut à six mois, puis, en I 824, une fille qui reçut le nom de Caroline. Les deux enfants, Gustave et sa sœur, presque du même âge, élevés ensemble, ne se quittaient guère ${ }^{22}$.

[Mme Flaubert] eut six enfants : Achille, l'aîné, rhumatisant, mais d'une santé robuste; deux garçons morts dans l'enfance; puis Gustave ; puis un garçon, mort dans la première enfance, et enfin Caroline ${ }^{23}$.

21. Caroline Commanville, "Souvenirs intimes", CEurres complètes de Gustave Flaubert. Correspondance, t. I, Conard, I926, p. XVI.

22. René Dumesnil, Gustave Flaubert, l'homme et l'cuvre, Desclée de Brouwer et Cie, ze édition, I 947, p. 34-36.

23. Ibid., p. 478 . 
En interprétant à sa guise ces informations un peu vagues et parfois fausses (nous verrons bientôt en quoi), Sartre établit sa propre version de l'histoire de la famille Flaubert : les parents de Gustave habitèrent rue du Petit-Salut pendant sept ans «entre i 8 I 2 et I 8 I 9 » (IDF, t. I, p. 84), ils y eurent trois garçons dont deux « moururent en bas âge » (IDF, t. I, p. 85), puis ils déménagèrent à l'Hôtel-Dieu où ils eurent encore trois enfants dont un seul mourut. Avec ce calcul en tête, Sartre condamne l'attitude de Mme Flaubert, qui se souvient de sa maison de la rue du Petit-Salut avec nostalgie. « Et voilà bien ce qui m'étonne : une seule mort prématurée suffit en général à plonger des parents dans le malheur; chez les Flaubert il s'en produit deux, coup sur coup : de quoi les ravager pour longtemps et leur faire prendre en horreur ce premier domicile. Or la vieille Mme Flaubert, trente ans plus tard, se plaît à revenir nostalgiquement dans la rue du Petit-Salut, à s'arrêter devant son ancien logement et à rappeler sans cesse qu'elle y a connu le bonheur » (IDF, t. I, p. 85 ). Sartre en conclut que le bonheur et le malheur de Caroline Flaubert ne dépendaient pas de ses enfants, mais d'une seule personne qui était Achille-Cléophas, son époux-père, et que Gustave ne pouvait être qu'un enfant mal-aimé que sa mère nourrissait ponctuellement mais sans affection.

Pourtant, le fait historique qu'ont établi des chercheurs plus précautionneux n'est pas le même. Lucien Andrieu nous donne une autre version, qui est la suivante :

Un ou deux ans plus tard, le nouveau prévôt d'anatomie trouva une petite maison au 8 de la rue du Petit-Salut. C'est là qu'il amena, le io février i 8 I 2, sa jeune épousée : Anne, Justine, Caroline Fleuriot [...]. C’est là que naquit, le 9 février I 8 I 3 , Achille Flaubert, le fils aîné, qui devait succéder un jour à son père. [...] Achille, Cléophas Flaubert fut donc contraint de rester 8, rue du Petit-Salut. Là devait naittre encore un enfant : Caroline, le 8 février I 8 6 , qui disparut vingt et un mois plus tard. Il semble alors que le père de Gustave, fatigué de traverser la ville tôt le matin et tard le soir, chercha à se rapprocher de son lieu de travail et vint, vers la fin de I 817 résider rue de Crosne-hors-la-Ville. Nous n'avons pu savoir l'endroit exact, il est vrai qu'il y resta si peu de temps puisqu'il perdit sa petite Caroline alors qu'il avait comme domicile I 3 , rue de Lecat. Là se trouve encore une petite énigme : s'agit-il d'un nouveau domicile ou tout simplement de l'Hôtel-Dieu, dont les numéros sont à notre connaissance 33, 5 I puis 55, rue de Lecat ? [...] D'un autre côté, lorsque naît un troisième enfant : Emile, Cléophas, le 8 novembre $18 \mathrm{I} 8$, qui meurt le $\mathrm{I} 3$ juillet $\mathrm{I} 8 \mathrm{I} 9$, puis un quatrième: Jules, Alfred, le 30 novembre I 8 1 9 , qui meurt le 29 juin I 822 , tous les actes de l'état civil portent comme adresse celle de l'Hôpital, ce qui est normal, Laumonier étant mort en janvier I 8 I 8, en février de la dite année, Achille, Cléophas Flaubert avait pris possession de l'appartement qui lui était destiné. [...] Gustave Flaubert naquit le I 2 décembre I 82 I et sa sœur Caroline, Joséphine, le I 5 juillet I $824^{24}$.

24. Lucien Andrieu, «Les maisons de la famille Flaubert dans la région rouennaise ", Les Amis de Flaubert, $\mathrm{n}^{\circ}$ 30, mai i 967, p. 9-10. 
Représentons ces deux versions dans un tableau qui nous permettra de voir plus clairement leur décalage.

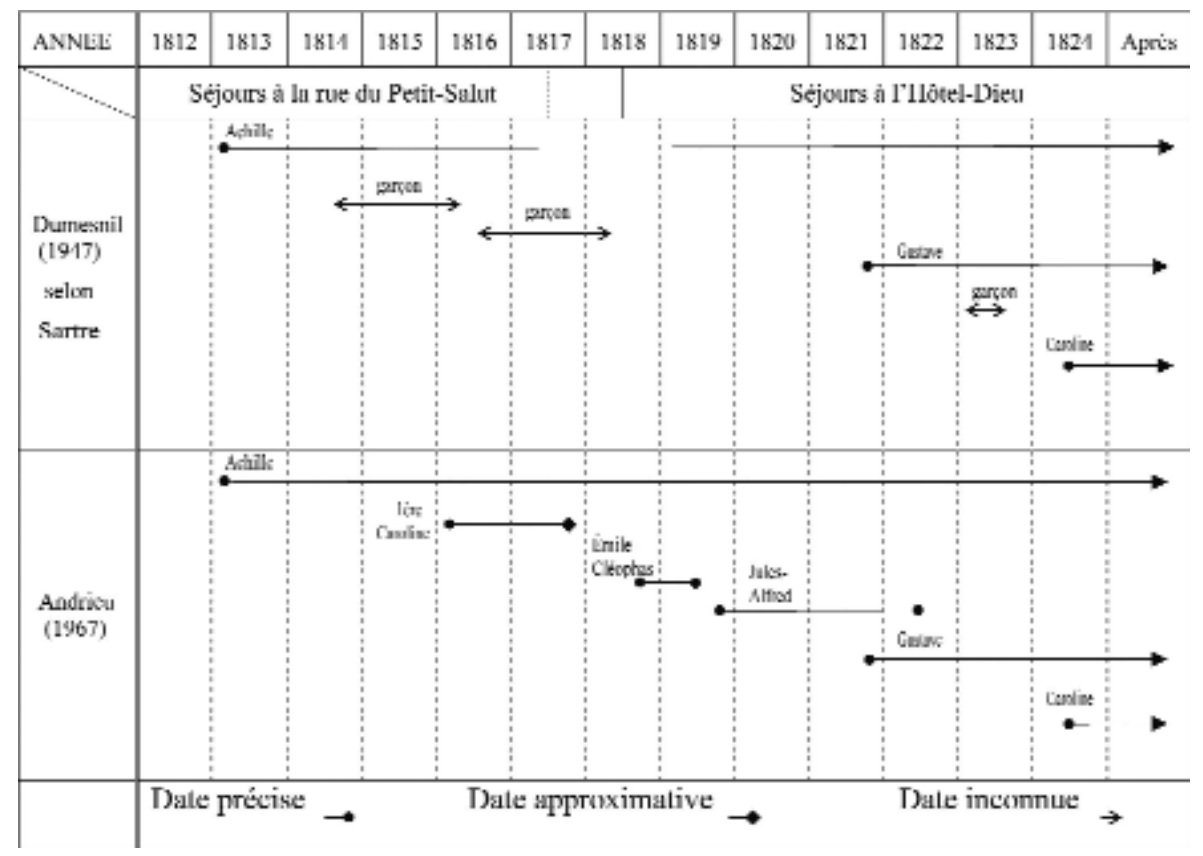

D'après l'investigation de Lucien Andrieu, lorsque la famille Flaubert quitta la maison de la rue du Petit-Salut vers la fin de 1817, ses membres comptaient quatre personnes, M. et Mme Flaubert, Achille, et une fille cadette. Contrairement à l'affirmation de Sartre, pendant les cinq ou six ans où ils habitèrent leur premier domicile, ils ne connurent aucun deuil et ce fut à l'Hôtel-Dieu ou à l'autre domicile à proximité de l'Hôtel-Dieu que M. et Mme Flaubert perdirent trois de leurs six enfants. Toute la thèse sartrienne concernant la mère de Gustave commence donc à vaciller. Les arguments sartriens soutenus pour justifier la thèse de « la passivité de Gustave conditionnée par le manque d'amour maternel » se sont désagrégés par la base, et ce sont surtout les raisonnements concernant « le planning familial » des parents de Gustave qui se dévoilent comme sans fondement.

Suivons ces arguments de Sartre en soulignant les parties qui ne correspondent pas aux faits réels et qui, néanmoins, sont utilisées par Sartre pour justifier ses hypothèses : Caroline et Achille-Cléophas « se marièrent en février i 8 I 2 et s'installèrent au 8 de la rue du Petit-Salut : ils devaienty rester sept ans. Mme Commanville écrit : "[...] Vois-tu, là se sont passées les meilleures années de ma vie." Pour nous, ce témoignage est de première importance. 
Sept ans de bonheur» (IDF, t. I, p. 84). Si l'on coupe en deux la vie conjugale de Mme Flaubert, comme elle nous y convie, « on remarque qu'elle eut, avant de s'établir à l'Hôtel-Dieu, trois fils dont un seul vivra ; après son établissement la proportion se renverse : des trois enfants qu'elle fait, un seul meurt» (IDF, t. I, p. 85 ). Si elle est tellement indifférente aux deux premiers décès de ses enfants, qu'elle peut se souvenir de son ancien logement avec un vrai bonheur, c'est parce qu'elle n'adore que son mari, comme père ressuscité qui récompense son enfance perdue. Après le déménagement, vient le commencement des années mauvaises : l'Hôtel-Dieu où les Flaubert « s'installent en 1819 » (IDF, t. I, p. I 29) est odieux pour Mme Flaubert et « lui découvre la très légère réserve d'un mari trop occupé. Pour la deuxième fois, Caroline est frustrée d'un père : elle retrouve sans le savoir, à travers cette retenue, les malheurs de son enfance solitaire [...]. Pour la première fois elle voulut une compensation; il n'y en avait qu'une. Une seule, rigoureusement définie par ses malheurs : une fille » (IDF, t. I, p. I 3 I). "Elle attendit treize ans cette chance qui vint trop tard. Treize ans pendant lesquels AcbilleCléophas lui fit cinq garçons. Le premier, elle l'accueillit avec plaisir [...] Mais, dès la seconde grossesse, elle commença d'attendre. Il y eut quatre déceptions : Gustave fut la troisième » (IDF, t. I, p. 90). Voici le planning familial de ce couple: « contre les petits mâles indiscrets qui se trompaient de ventre $[\ldots]$, une seule tactique : on efface tout et l'on recommence, aussi souvent qu'il le faudra, pour mettre au jour une petite fille et qui soit viable ». Cependant le couple devait se presser, sinon « les derniers venus seraient des enfants de vieux » (IDF, t. I, p. I 3 I ). Gustave est conçu à la fin du premier trimestre de I 82 I et « naît le I 2 décembre, un autre enfant le suit bientôt puis c'est Caroline en i 824. Cela signifie - on sevrait fort tard, à l'époque - que la mère de Gustave l'allaitait encore quand elle commença sa nouvelle grossesse et qu'il n'avait pas plus d'un an et demi quand son frère cadet disparut. Il a trois ans de plus que sa sœur, donc il faut que Mme Flaubert se soit trouvée enceinte, une fois encore, quand il était âgé de deux ans et quelques mois. Ainsi, depuis la naissance du futur écrivain jusqu'à sa troisième année, Mme Flaubert passe, presque sans transition, de la grossesse à l'accouchement, de l'allaitement au deuil, du deuil à la grossesse et à un nowvel accouchement. En neuf ans, trois enfants; trois enfants en moins de quatre : on passe de la nonchalance à la frénésie. [...] Trois en quatre ans, ces parents se hâtent, ils couchent ensemble pour avoir des enfants » (IDF, t. I, p. I 29). Et puis ils s'arrêtent nettement après la naissance de Caroline. Pourquoi ? «Eh bien, la raison est manifeste et je l'ai déjà dit : Mme Flaubert voulait une fille, quand elle l'eut, on tira le trait. Faut-il penser que Mme Flaubert avait déjà cette idée en tête quand le mari lui fit Gustave ? Je le crois » (IDF, t. I, p. I 30$)$. 
Ainsi, avant même sa naissance, Gustave est prédestiné par le planning familial de ses parents : il naitra cadet de sa famille, il nourrira pendant toute sa vie un sentiment d'infériorité par rapport à son inégalable frère aîné, il aura aussi une sœur cadette qui lui dérobera tout amour maternel, il ne pourra donc faire autrement qu'être un fils mal-aimé. Tout cela le constituera comme un sujet passif, le façonnera comme un idiot de la famille. Voilà la première moitié de la thèse de Sartre développée dans L'Idiot de la famille, qui est fondée sur ses hypothèses liées au «planning familial » du couple du docteur Flaubert. Mais il nous reste encore la seconde moitié de la thèse sartrienne, qui est soumise cette fois au «planning névrotique » de Gustave lui-même.

Examinons maintenant «le planning névrotique », par lequel Gustave, voué à la condition de cadet, donc à une condition inférieure, se décidera à «s'organiser en profondeur pour souffrir le moins possible » (IDF, t. I, p. I 86) de tous ces maux qui l'entourent. Conditionné par sa famille, constitué en tant que passivité et «mal vissé » dans le langage (IDF, t. I, p. 24), Gustave commence bien mal sa vie. Mais ce qu'on a fait de lui, Flaubert tente de le dépasser dans un lent mouvement de personnalisation, qui est organisé, selon Sartre, par le «planning névrotique » de Flaubert, ou par son « projet névrotique » (IDF, t. II, p. I930), étroitement lié à son « projet littéraire».

Exclu de l'univers actif et utilitariste des Flaubert, Gustave reprend à son compte la sentence familiale qui le condamne à l'inertie et il transforme ce verdict rendu par les autres en prétexte de sa stratégie névrotique, qui le dispensera enfin de la nécessité de prendre un état et lui donnera le droit de se vouer tranquillement à la littérature. Quand Gustave, à dix-sept ans, est mis en demeure de choisir une profession, il découvre son " être-bourgeois » $(I D F$, t. II, p. I485). Il le refuse, mais sa constitution passive lui interdit la révolte. Dans une apparente soumission, il va alors développer une névrose dont on verra les effets spectaculaires un jour de janvier i 844. Cette crise, que Flaubert a traversée entre dix-sept et vingt-deux ans, Sartre l'appelle "psychosomatique » (IDF, t. II, p. I 473). Pendant cette période de la crise psychosomatique, à laquelle Sartre accorde plus de trois cents pages sous le titre de « La prénévrose », Gustave prépare avec une «intention téléologique » (IDF, t. II, p. I 827 ) sa névrose qui se manifestera par la chute de I 844 à Pont-l'Évêque « comme réponse immédiate, négative et tactique à une urgence » (IDF, t. II, p. 1779). À partir de cette crise de Pont-l'Évêque, Gustave se retranche tranquillement à Croisset, où il mène une vie de séquestration volontaire en maintenant une tension religieuse pour trouver un style qui lui permette d'atteindre l'Idéal. Il deviendra un martyr souffrant par la tâche douloureuse d'écrire, par une recherche désespérée de la Beauté. 
Telle est la version sartrienne du "planning névrotique » de Flaubert, où Sartre a essayé de nous montrer le «mouvement dialectique par lequel le projet artistique et le projet névrotique se conditionnent mutuellement, au point que l'écriture devient névrose et la névrose littérature » (IDF, t. II, p. I930). Pourtant cette thèse de Sartre repose avant tout sur un diagnostic assez douteux concernant la nature de la maladie de Flaubert: Sartre a la conviction que Gustave Flaubert souffre de névrose, et non d'épilepsie.

Névrose ? Ou épilepsie? On a beaucoup écrit sur la nature de cette maladie. Mais quelle différence existe-t-il entre ces deux dénominations ? La détermination de la maladie d'un écrivain mort il y a plus d'un siècle peut-elle être un sujet si important? Oui, du moins pour Sartre : parce que l'enjeu de cette querelle n'est autre que la liberté d'un individu, thème essentiel de la philosophie sartrienne ${ }^{25}$. Qu'est-ce que la névrose ? Sa définition même n'est pas si simple. Dans leur Vocabulaire de la psychanalyse, Jean Laplanche et JeanBaptiste Pontalis nous donnent, à titre d'exemple, cet essai de définition tiré d'un manuel de psychiatrie : "Ce sont les troubles des conduites, des sentiments ou des idées qui manifestent une défense contre l'angoisse et constituent à l'égard de ce conflit interne un compromis dont le sujet tire dans sa position névrotique un certain profit ${ }^{26} \gg$.

Contrairement à «l'épilepsie » qui est une des affections « pour lesquelles on a de bonnes raisons de supposer l'existence d'une lésion du système ner$v^{2} x^{27}$ », «la névrose » est définie pathologiquement comme un « trouble fonctionnel du système nerveux sans lésion organique de celui-ci, se manifestant par des troubles physiques au niveau d'un organe ${ }^{28} »$. Si Flaubert a souffert d'épilepsie, maladie causée par des défauts organiques, toutes les conséquences de cette maladie, sa vie et ses œuvres, ne sont que les produits du hasard, c'est-à-dire que, dans l'optique sartrienne, il y a peu de place pour la liberté de l'homme. Ce diagnostic aboutit à la thèse du déterminisme organique dans laquelle l'homme n'est plus maître de son propre destin. Si l'événement de janvier I 844 fut le résultat d'une épilepsie, toute l'analyse de la protohistoire de Flaubert et de ses œuvres de jeunesse s'effondre, tout l'argument sartrien du mouvement dialectique entre l'Esprit objectif et l'individu perd son fondement.

Pour Sartre, la crise de Pont-l'Évêque doit être le fruit d'une intention et le résultat d'un choix de vie. Selon lui, Flaubert choisit donc la maladie pour

25. Voir IDF, t. II, p. 2 I 50 : «La maladie de Gustave exprime dans sa plénitude ce qu’il faut bien appeler sa liberté».

26. J. Laplanche et J.-B. Pontalis, Vocabulaire de la psychanalyse, PUF, I967, p. 270.

27. Ibid., p. 268. Nous soulignons.

28. Trésor de la langue française, Gallimard, I986. Nous soulignons. 
mieux devenir cette sorte d'écrivain qu'il devait se déterminer à être, puisque cet « engagement hystérique » (IDF, t. II, p. I 864) lui permet d'accomplir son projet névrotique, et de justifier ainsi sa séquestration intentionnelle tout comme les conversions symboliques qu'il sut imposer à son mal en relation avec la «névrose objective » et la programmation propre à son temps. Pour étayer tous ces arguments, Sartre doit prouver à tout prix que Flaubert était névrosé, et non épileptique. Le diagnostic du docteur René Dumesnil avec sa « démonstration très convaincante » (IDF, t. II, p. 1796) lui fournit une bonne preuve pour sa thèse. Pourtant, à l'époque où Sartre écrit son livre, la plupart des chercheurs flaubertiens optent plutôt pour l'épilepsie.

La nature de la maladie nerveuse de Flaubert fit couler beaucoup d'encre dès i 860. Les contemporains de Flaubert, Maxime Du Camp, Henry Monnier, les Goncourt, le docteur Georges Pouchet, diagnostiquaient déjà l'épilepsie ${ }^{29}$. Mais René Dumesnil, qui avait écrit, vers 1905, une thèse de médecine sur la maladie de Flaubert, a réexaminé les témoignages des proches de Flaubert et sa correspondance, et il a tenté de prouver que Flaubert ne souffrait que d'une névrose. La base principale de ses analyses est avant tout la description des crises faite par Du Camp dans ses Souvenirs littéraires ${ }^{30}$, et par Flaubert lui-même sous forme d'extraits de sa correspondance ${ }^{31}$. Dans l'article intitulé : « La maladie et la mort de Flaubert», le docteur Dumesnil a essayé d'écarter le diagnostic de l'épilepsie, opinion généralement adoptée depuis la publication des Souvenirs littéraires, et il a officialisé à son tour le diagnostic de la névrose. Voici des extraits de son article :

Ce qu'on vient de lire ne s'applique absolument pas à l'épilepsie essentielle, au mal sacré. Et voici les raisons qui font écarter formellement ce diagnostic [...]. Pas de relâchement des sphincters pendant la crise, pas de morsure de la langue. Si ces symptômes avaient existé, Du Camp aurait été trop heureux de pouvoir les rapporter. [...] Mais encore l'âge - vingt-deux ans - auquel survient la première crise, fait écarter a priori l'épilepsie essentielle, maladie qui se manifeste presque toujours dès l'enfance. Enfin jamais un épileptique n'a le temps de choisir l'endroit où il tombe, d'aller s'étendre dans son lit même, « comme il se serait couché tout vivant dans un cercueil ». Et plus tard, quand il parle de ses crises, Flaubert est tout aussi précis : « Toujours j'avais conscience, même quand je ne pouvais plus parler ", écrit-il à Louise Colet. "L'être raison allait jusqu'au bout, sans cela la souffrance eût été nulle ». Où est l'épileptique qui garde conscience de ses crises, qui peut en exposer les phases ${ }^{22}$ ?

29. Voir Jean Bruneau, Les Débuts littéraires de Gustave Flaubert, Armand Colin, I962, p. 360, note io.

30. Maxime Du Camp, Souvenirs littéraires, Aubier, r 994, p. 199-203.

3 I. Principalement, la lettre à Ernest Chevalier du i er février i 844 et la lettre à Louise Colet du 2 septembre 1853.

32. René Dumesnil, Gustave Flaubert, l'homme et l'auvre, éd. citée, Appendice A, p. 487-489. Nous soulignons. 
C’est l'avis que Sartre a totalement adopté. Après avoir longtemps discuté sur la nature de la maladie de Flaubert, il opte pour la névrose, à moins qu’il n'ait commencé dès le début par l'hypothèse de la névrose pour parvenir à cette conclusion. Cependant la médecine contemporaine n'est pas d'accord avec ce diagnostic. En i 962, le docteur Galérant publie un compte rendu de la thèse du docteur Gallet, qui propose l'épilepsie pour définir la maladie de Flaubert, en répondant point par point aux arguments du docteur Dumesnil :

La conclusion du docteur Gallet est formelle : épilepsie, sans aucun doute. [...] Le docteur Dumesnil a nié l'épilepsie sous prétexte que :

I $^{\circ}$ L'aura est de trop longue durée et l'épileptique ne choisit pas l'endroit où il va tomber. Or, Flaubert allait s'étendre sur son divan au moment où il allait entrer dans la phase convulsive. C'est une erreur, rétorque le jeune confrère à son ancien; il ne faut pas confondre deux éléments distincts : les prodromes et l'aura. Or, nous savons que beaucoup d'épileptiques sont avertis parfois plusieurs jours à l'avance de l'imminence d'une crise. Flaubert disposait de quelques minutes d'intervalle libre, c'était suffisant pour aller s'étendre sur un divan.

$2^{\circ}$ Il garde le sowvenir de sa crise. Autre erreur d'interprétation. Il gardait le souvenir de l'aura, ce qui est normal, mais pas de la crise. [...]

$3^{\circ}$ L'épilepsie ne commence pas à 22 ans. C'est faux. Cette affection commence à n'importe quel âge, selon les causes qui la déterminent.

$4^{\circ}$ Il n'y avait pas d'incontinence d'urine au cours de la crise, sinon Du Camp aurait été trop beureux de le dire. C'est là une affirmation purement gratuite et qui, nous l'avons vu, ne résiste pas à l'examen ${ }^{33}$.

Le docteur Gallet et le docteur Galérant justifient leurs arguments en levant le doute sur l'authenticité du témoignage de Du Camp. Même s'il est vrai que Du Camp est « un témoin douteux et qui commence par se tromper de date et de lieu» (IDF, t. II, p. I784), son témoignage est, selon eux, techniquement «insoupçonnable ${ }^{34} »$. L'ÉPILEPSIE. C'est ce à quoi conclut aussi la médecine anglophone. Le docteur Arthur Ecker, professeur de neurochirurgie à la Faculté de médecine de l'Université Syracuse, qui a indépendamment mené son analyse sur le cas de Flaubert, aboutit à une conclusion similaire ${ }^{35}$. Depuis ces confirmations de la médecine d'aujourd'hui, le diagnostic de l'épilepsie est généralement admis chez les critiques flaubertiens ${ }^{36}$.

33. Docteur Galerant, «Quel diagnostic aurions-nous fait si nous avions soigné Gustave Flaubert? - Notes pour l'analyse de l'ouvrage du Docteur Gallet ", Les Amis de Flaubert, ${ }^{\circ}{ }^{20}$, I962, p. 7-8.

34. Docteur Galérant, «Flaubert vu par les médecins d'aujourd'hui », Europe, septembrenovembre 1969, p. 108.

35. Voir Benjamin F. Bart, Flaubert, New York, Syracuse University Press, I 967, p. 75 2-753.

36. Voir Jean Bruneau, "Notes et variantes ", dans Flaubert, Correspondance I (I830-I85I), Gallimard, "Bibliothèque de la Pléiade », I973, p. 943-944, et Marthe Robert, En haine du roman, Balland, «Le Livre de Poche », I982, p. 57-68. 
Alors, Sartre n'était-il pas au courant de tout cela ? Peut-être que si. Mais ce diagnostic de l'épilepsie qui ne considère la maladie de Flaubert que comme un accident, n'a pas de sens pour lui. Cette sorte de déterminisme organique ne mène qu'à réduire l'univers singulier créé par Flaubert au simple résultat accidentel de ses défauts physiques, comme le montre, par exemple, l'article de Philippe Bonnefis, où l'auteur voit toute l'œuvre de Flaubert comme l'aboutissement involontaire d'un épileptique, déclarant: «L'épilepsie qui illuminait Flaubert et le saoulait de couleurs était un mal divin, un sacre, une consécration ${ }^{37} \gg$.

Pour Sartre, d'origine organique ou non, le mal de Flaubert «s'est organisé en fonction d'une intention originelle ; sa structuration foudroyante, à Pontl'Évêque, n'est pas un fait accidentel mais une nécessité pourvue de sens» (IDF, t. II, p. 1796). Sartre y voit une finalité, une « stratégie positive » (IDF, t. II, p. 1933) qui permet à Flaubert de se redresser dans son existence difficile. Au départ, il y a un être humain jeté dans le monde et assailli par « le praticoinerte » de son environnement. Cet être humain, en tant qu'être-liberté au plus profond de son être, a choisi de se faire passif définitivement dans le monde par sa maladie ; et ce sera ce choix volontaire qui fera que Gustave deviendra Gustave Flaubert, l'écrivain. Bref, la crise de i 844 de Flaubert est "l'aboutissement mathématique de sa malheureuse jeunesse » et «son génie n'est que la conséquence mathématique de sa fausse mort à Pont-l'Évêque, désespoir poussé à l'extrême » (IDF, t. II, p. 2024). À ce niveau-là, nous pourrions dire que l'essai biographique de Sartre sur Flaubert frôle le roman. Bien entendu, pour Sartre, cette hypothèse de la névrose est aussi un choix. Flaubert aurait été sans doute épileptique, et contrairement à ce que Sartre avance, l'épilepsie n'aurait pas l'hystérie pour origine. Peu importe. Ce qui a intéressé Sartre, ce n'est pas l'exactitude historique de la vie de Flaubert, mais surtout la recherche méthodologique pour atteindre à une vérité, à la compréhension totale d'un "universel singulier ", à la cohérence d'un individu nommé Gustave Flaubert.

Nous venons ainsi d'examiner L'Idiot de la famille sous un angle plutôt positiviste, et nous constatons que les regards sceptiques des flaubertiens à l'égard du «Flaubert» sartrien ne sont pas sans raison. Comme Sartre l'a dit le lendemain de la parution des deux premiers tomes de L'Idiot de la famille, ce qu'il a dépeint dans son ouvrage n'est pas Flaubert tel qu'il fut, mais «Flaubert tel qu[il] l'imagine ${ }^{38} »$. Par là, ce livre, en requérant de nous plutôt l'imagination

37. Ph. Bonnefis, «Aura epileptica », Magazine littéraire, $\mathrm{n}^{\circ}$ 250, février I988, p. 4I.

38. J.-P. Sartre, Situations $X$, éd. citée, p. I I 4. 
romanesque que l'investigation historique, ressortit moins à la critique savante qu'à la pure littérature. Mais nous savons qu'en lisant L'Idiot de la famille comme un roman ou une autobiographie déguisée, nous pourrons en apprécier plus justement la valeur. Et la question de savoir si L'Idiot de la famille relève du roman ou d'une (auto)biographie gagnerait sans doute à être éclairée par le débat général sur les rapports entre mythe et histoire, qui a été suscité surtout par les positions de l'herméneutique. Il apparait que les deux récits ne sont pas toujours aussi aisés à différencier. La question se trouve aussi de plus en plus posée de savoir si la fiction ne peut être un moyen adéquat pour explorer le réel. Bref, même s'il est vrai que L'Idiot de la famille n'est pas un livre très utile pour les flaubertistes, ce livre est sans doute, comme dit J. Bruneau, « extrêmement important pour tout le monde 39 ».

39. Jean Bruneau, «L'intervention », Langages de Flaubert, éd. citée, p. 230. 